\title{
Severe Bradycardia Induced by Sofosbuvir and Amiodarone which Resolved after the Discontinuation of Both Drugs
}

\author{
Chikako Monoe $^{1}$, Hiroyasu Shimizu ${ }^{1}$, Kanae Kitaguchi ${ }^{2}$ and Hiraku Funakoshi ${ }^{1}$
}

\begin{abstract}
:
Amiodarone, prescribed for cardiac conditions, including dysrhythmia, is associated with bradycardia. However, few reports have demonstrated that bradycardia is observed when amiodarone is used in combination with sofosbuvir, a direct-acting antiviral drug for chronic hepatitis $\mathrm{C}$. We herein report the case of a male patient in his 70s with chronic atrial fibrillation and prostatic hyperplasia and was also taking amiodarone, who experienced severe bradycardia and syncope a day after taking sofosbuvir and ribavirin for chronic hepatitis C. After discontinuing amiodarone, ribavirin, and sofosbuvir, bradycardia did not recur. Therefore, we must consider drug interactions between amiodarone and sofosbuvir in patients with severe bradycardia.
\end{abstract}

Key words: amiodarone, bradycardia, drug interaction, sofosbuvir

(Intern Med 59: 2619-2622, 2020)

(DOI: 10.2169/internalmedicine.4488-20)

\section{Introduction}

Hepatitis C virus (HCV) causes both acute and chronic hepatitis, and many patients develop chronic infection (1). In 2014, interferon-free drugs and direct-acting antivirals (DAAs), such as sofosbuvir, were introduced into the clinical setting.

Amiodarone is an antiarrhythmic drug classified as a group III agent (2). Long-term oral amiodarone has many side effects due to its accumulation in tissues, which is generally not observed with short-term intravenous therapy (3). We herein report the case of a male patient in his 70s with chronic atrial fibrillation, chronic hepatitis $\mathrm{C}$, and prostatic hyperplasia who experienced severe bradycardia and syncope after the administration of sofosbuvir along with amiodarone.

\section{Case Report}

A man in his 70s visited our hospital suffering from extreme bradycardia and syncope. He was being treated for chronic atrial fibrillation, chronic hepatitis $\mathrm{C}$, and prostatic hyperplasia by his primary care doctor. He was taking amio- darone for atrial fibrillation because he had undergone defibrillation twice despite receiving standard therapy. His electrocardiogram (ECG) which had been previously checked by his primary care doctor showed atrial fibrillation (Fig. 1). He had previously received appropriate therapy for cirrhosis, however, his liver function gradually worsened, and he was prescribed sofosbuvir and ribavirin. On the second day of the new therapy, he experienced a transient loss of consciousness (LOC) and collapsed approximately one hour after taking the sofosbuvir. The patient was concerned and visited the Emergency Department for consultation.

On physical examination, he was conscious, and his vital signs were as follows: temperature, $36.6^{\circ} \mathrm{C}$; heart rate, 38 beats/minute; blood pressure, $139 / 76 \mathrm{mmHg}$; respiratory rate, 16 breaths/minute; and oxygen saturation, $99 \%$ on room air. Auscultation of the lungs and heart was normal. The sensation and muscle strength in both the arms and legs were intact. Blood tests showed no obvious abnormalities (Table). An electrocardiogram showed only sinus bradycardia (Fig. 2). Chest radiography and chest and head computed tomography (CT) were normal. Abdominal CT revealed liver hemochromatosis, but the findings were otherwise clear. Transthoracic echocardiography showed a left ventricular ejection fraction of $60 \%$, an absence of asynergy,

${ }^{1}$ Department of Emergency and Critical Care Medicine, Tokyobay Urayasu Ichikawa Medical Center, Japan and ${ }^{2}$ Department of General Internal Medicine, Tokyobay Urayasu Ichikawa Medical Center, Japan

Received: January 23, 2020; Accepted: April 28, 2020; Advance Publication by J-STAGE: June 30, 2020

Correspondence to Dr. Hiraku Funakoshi, hfunakoshi-tbmc@umin.org 


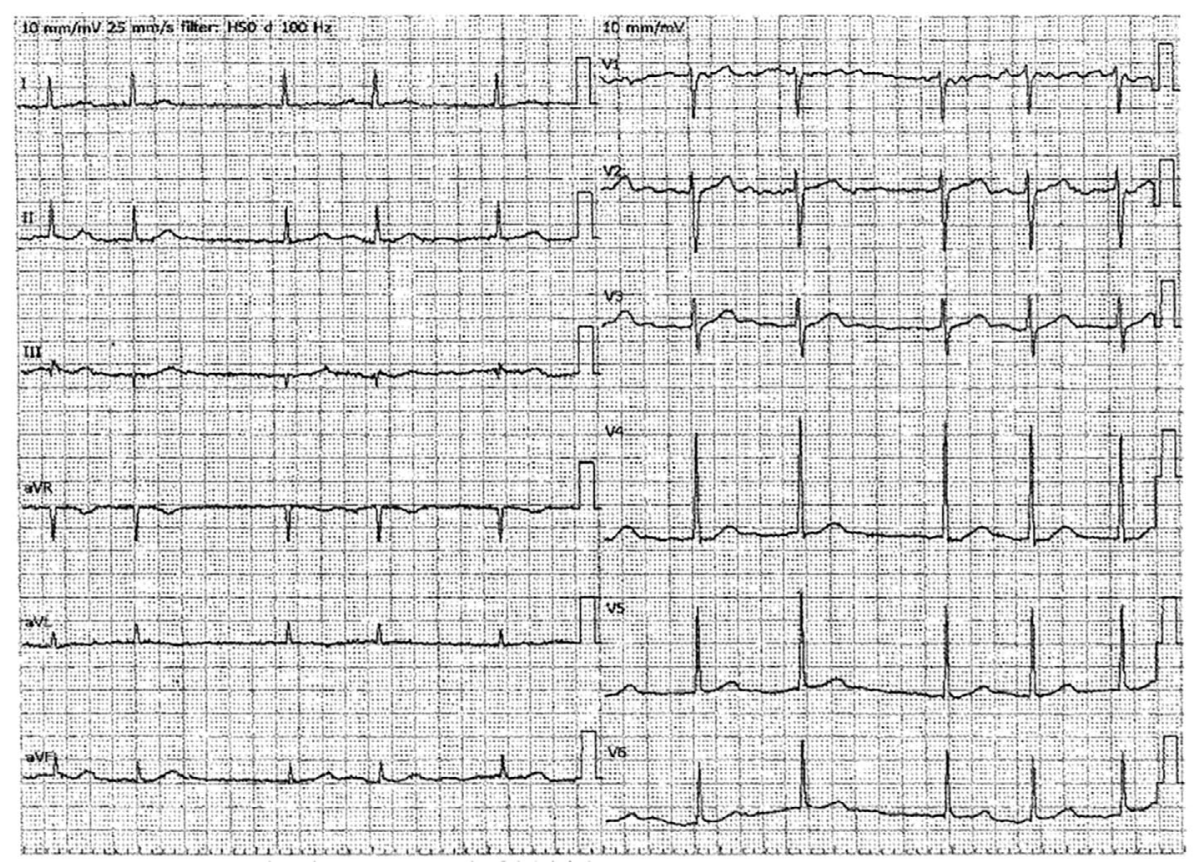

Figure 1. An electrocardiogram checked by the patient's primary care doctor. The electrocardiogram showed atrial fibrillation.

Table. Results of Blood Examination of the Patient.

\begin{tabular}{lclrlc}
\hline Parameter & Value & Parameter & Value & Parameter & Value \\
\hline Alb & $3.0 \mathrm{~g} / \mathrm{dL}$ & T-Bil & $0.42 \mathrm{mg} / \mathrm{dL}$ & CRP & $1.54 \mathrm{mg} / \mathrm{L}$ \\
UN & $19.8 \mathrm{mg} / \mathrm{dL}$ & Glu & $111 \mathrm{mg} / \mathrm{dL}$ & TSH & $4.570 \mu \mathrm{IU} / \mathrm{mL}$ \\
CRE & $0.77 \mathrm{mg} / \mathrm{dL}$ & $\mathrm{Na}$ & $140 \mathrm{mEq} / \mathrm{L}$ & FT4 & $1.51 \mathrm{ng} / \mathrm{dL}$ \\
AST & $216 \mathrm{U} / \mathrm{L}$ & $\mathrm{K}$ & $4.2 \mathrm{mEq} / \mathrm{L}$ & $\mathrm{WBC}$ & $4,600 / \mu \mathrm{L}$ \\
ALT & $201 \mathrm{U} / \mathrm{L}$ & $\mathrm{Cl}$ & $105 \mathrm{mEq} / \mathrm{L}$ & $\mathrm{Hb}$ & $10.9 \mathrm{~g} / \mathrm{dL}$ \\
ALP & $298 \mathrm{U} / \mathrm{L}$ & $\mathrm{Ca}$ & $7.4 \mathrm{mg} / \mathrm{dL}$ & Plt & $22.4 \times 10^{4} / \mu \mathrm{L}$ \\
LDH & $337 \mathrm{U} / \mathrm{L}$ & $\mathrm{P}$ & $3.2 \mathrm{mg} / \mathrm{dL}$ & & \\
$\gamma$-GTP & $112 \mathrm{U} / \mathrm{L}$ & $\mathrm{Mg}$ & $2.1 \mathrm{mg} / \mathrm{dL}$ & & \\
Amiodarone & $0.893 \mu \mathrm{gg} / \mathrm{mL}[$ normal range: $0.5-1.0 \mu \mathrm{gg} / \mathrm{mL}]$ & & \\
\hline
\end{tabular}

Alb: albumin, UN: urea nitrogen, CRE: creatinine, AST: aspartate aminotransferase, ALT: alanine aminotransferase, ALP: alkaline phosphatase, LDH: lactate dehydrogenase, $\gamma$-GTP: $\gamma$-glutamyl transpeptidase, TBil: total bilirubin, Glu: glucose, Na: sodium, K: potassium, $\mathrm{Cl}$ : chloride, Ca: calcium, P: phosphorus, Mg: magnesium, CRP: C-reactive protein, TSH: thyroid stimulating hormone, FT4: free thyroxin, WBC: white blood cell, Hb: hemoglobin, Plt: platelet

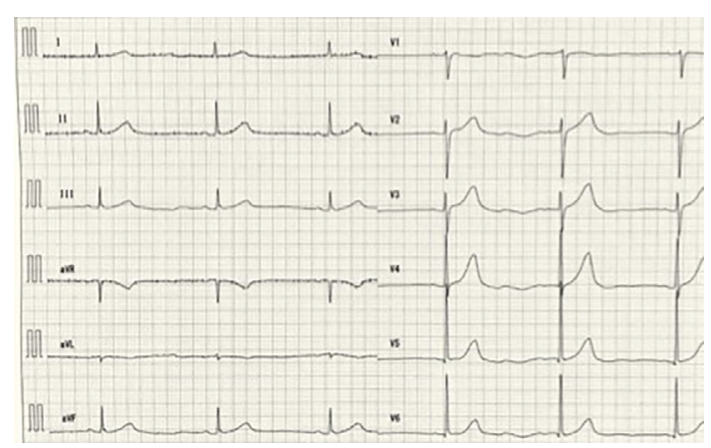

Figure 2. An electrocardiogram performed at the emergency department. The electrocardiogram shows sinus bradycardia without axis deviation and ST-T changes. QTc is $\mathbf{5 0 0}$ milliseconds. and opening restrictions of the aortic valve.

The patient's medication history included oral ursodeoxycholic acid (300 mg, >10 years), candesartan cilexetil+amlodipine besilate ( $4 \mathrm{mg},>10$ years), dabigatran (220 mg, 3 years), amiodarone (200 mg, 3 years), sofosbuvir (400 mg, 2 days), and ribavirin (200 mg, 2 days).

He was hospitalized for observation. We suspected that drug-induced bradycardia was the cause of the syncope and discontinued the amiodarone, sofosbuvir, and ribavirin after admission. Bradycardia and syncope were not observed during the hospital stay, and he was discharged on day 9. No further bradycardia symptoms were reported at follow-up visits for one year.

The patient provided his written informed consent for the publication of this case. 


\section{Discussion}

We herein describe the case of an elderly male patient treated with amiodarone for chronic atrial fibrillation who presented with extreme bradycardia and syncope after taking sofosbuvir and ribavirin for hepatitis $\mathrm{C}$. The only abnormality observed was bradycardia, which resolved after the discontinuation of amiodarone, sofosbuvir, and ribavirin, with no further recurrence.

Following HCV infection, approximately $70 \%$ of patients experience a state of persistent infection (1). The goal of HCV treatment is to eradicate HCV RNA. Sofosbuvir, a nucleic acid-type NS5B polymerase inhibitor, is a novel drug for the treatment of hepatitis $\mathrm{C}$, which, like other DAAs, has replaced interferons.

Our patient experienced transient LOC after taking the new medications. The possible causes of transient LOC are generally grouped into four major categories: reflex syncope, orthostatic syncope, cardiac dysrhythmias, and structural cardiopulmonary disease. We presumed that the cause of the patient's LOC was cardiac dysrhythmia and extreme sinus bradycardia.

Sinus bradycardia is seen in many pathophysiological settings, including sick sinus syndrome, medication effects, and acute myocardial infarction (4-7). The bradycardia, in this case, appears to have been drug-induced as symptoms indicative of other causes were absent, and the bradycardia disappeared after the discontinuation of amiodarone and sofosbuvir.

To date, nine reports have described bradycardia caused by the interaction between amiodarone and sofosbuvir. In 2015, the US Food and Drug Administration issued a safety announcement regarding a prominent slowing of the heart rate when amiodarone is combined with sofosbuvir (8). Renet et al. reported two cases of extreme bradycardia after the first doses of sofosbuvir and daclatasvir in patients receiving amiodarone (9).

One was a woman in her 60 s with chronic hepatitis, persistent atrial fibrillation, hypertension, and dyslipidemia. She had a medical history of ischemic stroke, basilar artery aneurysm rupture, and pulmonary embolism. On day 1, she took sofosbuvir and daclatasvir for the first time in addition to her usual medications (amiodarone, atenolol, atorvastatin, and fluindione). Thirty minutes later, she lost consciousness. It happened several times. At the hospital, she suffered cardiac arrest and underwent cardiopulmonary resuscitation, and spontaneous circulation was achieved. A normal sinus rhythm was achieved, and she was admitted to the intensive care unit. After amiodarone, sofosbuvir, and daclatasvir were stopped, she had no further loss of consciousness and her ECG returned to normal.

The second case was that of a man in his 50s with chronic HCV infection, cirrhosis, diabetes mellitus, and paroxysmal atrial fibrillation. Two hours after starting sofosbuvir and daclatasvir therapy in addition to his usual medica- tions (amiodarone, propranolol and fluindione), he experienced syncope. After being hospitalized, his ECG showed sinus node dysfunction ( 27 beats/minute). After the intravenous administration of atropine $(1 \mathrm{mg})$, he recovered a normal sinus rhythm. Amiodarone and propranolol were stopped, and the patient was kept on sofosbuvir and daclatasvir therapy until day 4 , during which time sinus bradycardia was recorded 2 hours after he took sofosbuvir and daclatasvir. On day 5, he stopped the two drugs, and bradycardia did not recur.

Our findings suggest that a plausible mechanism was Pglycoprotein (P-gp) inhibition by amiodarone. P-gp, also known as multidrug resistance protein 1 or ATP-binding cassette sub-family B member 1 , is a prominent protein of the cell membrane that pumps foreign substances out of cells via an ATP-dependent mechanism. P-gp also transports substrates, such as chemotherapeutic agents, antiarrhythmic drugs, lipids, steroids, xenobiotics, peptides, bilirubin, cardiac glycosides such as digoxin, immunosuppressive agents, and HIV-type 1 antiretroviral therapy agents, across the cell membrane. Sofosbuvir is a P-gp substrate. Thus, the presence of a therapeutic amiodarone concentration leads to increased sofosbuvir absorption. With P-gp also present on cardiomyocytes, this efflux transporter usually prevents the cellular uptake of sofosbuvir. However, when used in combination with amiodarone, which has a P-gp inhibitory action, this protective effect could be blocked, sofosbuvir concentrations would increase in cardiomyocytes, and bradycardia could occur (9).

As in previous cases, this patient had bradycardia shortly after taking sofosbuvir. The blood concentrations of sofosbuvir peak 0.5 hours after ingestion, so the onset of symptoms was thought to be early. When stopping sofosbuvir for the treatment of bradycardia, it is more effective to discontinue the new drug than to discontinue amiodarone, given the half-life of both drugs. Therefore, if amiodarone cannot be discontinued, then the treatment can be expected to be effective by stopping sofosbuvir alone.

In Japan, atrial fibrillation and hepatitis $\mathrm{C}$ are rarely treated by the same physician. In addition, because amiodarone is not approved for supraventricular tachycardia by insurance in Japan, a hepatologist will not expect patients with atrial fibrillation to be taking amiodarone unless the patients accurately report their medical history by themselves. Due to potentially severe complications caused by drug interactions such as bradycardia, as seen in this patient, when starting sofosbuvir, patients' medication history must be accurately recorded.

In summary, when encountering patients who have been prescribed sofosbuvir and amiodarone, the emergency department physician must be mindful that the interaction between these two drugs could result in bradycardia or LOC.

The authors state that they have no Conflict of Interest (COI). 


\section{References}

1. Drafting Committee for Hepatitis Management Guidelines, the Japan Society of Hepatology. JSH guidelines for the management of hepatitis C virus infection: a 2014 update for genotype 1. Hepatol Res 44: 59-70, 2014.

2. Zimetbaum P. Amiodarone for atrial fibrillation. N Engl J Med 356: 935-941, 2007.

3. Desai AD, Chun S, Sung RJ. The role of intravenous amiodarone in the management of cardiac arrhythmias. Ann Intern Med 127: 294-303, 1997.

4. Linzer M, Yang EH, Estes NA 3rd, Wang P, Vorperian VR, Kapoor WN. Diagnosing syncope. Part 1: value of history, physical examination, and electrocardiography. Clinical Efficacy Assessment Project of the American College of Physicians. Ann Intern Med 126: 989-996, 1997.

5. Calkins H, Shyr Y, Frumin H, Schork A, Morady F. The value of the clinical history in the differentiation of syncope due to ventricular tachycardia, atrioventricular block, and neurocardiogenic syncope. Am J Med 98: 365-373, 1995.

6. Moya A. Taskforce for the diagnosis and management of syncope;
European Society of Cardiology (ESC); European Heart Rhythm Association (EHRA); Heart Failure Association (HFA); Heart Rhythm Society (HRS). Guidelines for the diagnosis and management of syncope (version 2009). Eur Heart J 30: 2631-2671, 2009.

7. Shen WK, Sheldon RS, Benditt DG, et al. 2017 ACC/AHA/HRS guideline for the evaluation and management of patients with syncope: a report of the American College of Cardiology/American Heart Association Task Force on Clinical Practice Guidelines and the Heart Rhythm Society. J Am Coll Cardiol 70: e39-e110, 2017.

8. Back DJ, Burger DM. Interaction between amiodarone and sofosbuvir-based treatment for hepatitis $\mathrm{C}$ virus infection: potential mechanisms and lessons to be learned. Gastroenterology 149: 1315-1317, 2015.

9. Renet S, Chaumais MC, Antonini T, et al. Extreme bradycardia after first doses of sofosbuvir and daclatasvir in patients receiving amiodarone: 2 cases including a rechallenge. Gastroenterology 149: 1378-1380, 2015.

The Internal Medicine is an Open Access journal distributed under the Creative Commons Attribution-NonCommercial-NoDerivatives 4.0 International License. To view the details of this license, please visit (https://creativecommons.org/licenses/ by-nc-nd/4.0/).

(C) 2020 The Japanese Society of Internal Medicine

Intern Med 59: 2619-2622, 2020 